\title{
STEREODYNAMICAL STUDIES OF VELOCITY ALIGNED PHOTOFRAGMENTS
}

\author{
M. BROUARD, S. P. DUXON, P. A. ENRIQUEZ, R. SAYOS $\dagger$ \\ and J. P. SIMONS \\ Chemistry Department, The University, Nottingham NG7 2RD, UK
}

\begin{abstract}
The state resolved stereodynamics of bimolecular reactions can be probed using velocity aligned photofragments as reagents, and polarised, Doppler resolved laser detection techniques for the products. The new strategy and its application to the reaction $\mathrm{O}\left({ }^{1} \mathrm{D}\right)+\mathrm{N}_{2} \mathrm{O} \rightarrow \mathrm{NO}+\mathrm{NO}$ are outlined.
\end{abstract}

KEY WORDS: Stereodynamics, vector correlations, photodissociation.

\section{INTRODUCTION}

Polarised laser probing allows unprecedented levels of reagent state specification/ selection and product state resolution and, with very narrow line lasers, of product velocity resolution via Doppler resolved spectroscopic techniques. ${ }^{1-4}$ Such methods, first initiated for the stereodynamics of photodissociation, have now been extended to create a new experimental strategy for probing the stereodynamics of $b i$-molecular reactions. ${ }^{5-7}$ Polarised photodissociation is used to generate superthermal "beams" of velocity aligned photofragments and the state-to-state stereodynamics of their subsequent collisions are probed at high resolution and sensitivity in a "simple" bulb experiment. The new strategy both complements and amplifies the more conventional molecular beam scattering technique. Doppler resolution of the polarised laser induced fluorescence (or ionisation) spectrum of the nascent $b i$-molecular collision products can allow determination of their quantum state distributions, scalar pair correlations and angular vector correlations, referenced initially to the laboratory frame, but after a simple laboratory to centre of mass transformation, to the $b i$-molecular collision frame.

The highly exothermic reaction

$$
\mathrm{O}\left({ }^{1} \mathrm{D}\right)+\mathrm{N}_{2} \mathrm{O} \rightarrow \mathrm{NO}+\mathrm{NO}, \Delta E_{\mathrm{O}}=341 \mathrm{~kJ} \mathrm{~mol}^{-1}
$$

which follows the photodissociation of $\mathrm{N}_{2} \mathrm{O}$ at $193 \mathrm{~nm}$ leads to the generation of rotationally excited and aligned NO molecules. The excitation into all vibrational levels up to and beyond the thermo-chemical limit of $v_{\mathrm{NO}}=16$ establishes the

\footnotetext{
$\dagger$ Present address: Departamento di Quimica Fisica, Universidad de Barcelona, 08028, Barcelona, Spain.
} 
primary generation of superthermal, velocity aligned $\mathrm{O}\left({ }^{1} \mathrm{D}\right)$ atoms. ${ }^{17}$ The current status of our understanding of the reaction follows a brief outline of the basic theory for velocity aligned photofragment dynamics.

\section{THEORY}

Translation Alignment. Figure 1 shows the Newton diagram for the collision of a superthermal velocity aligned atom A with a stationary target molecule $\mathrm{BC}$. The LAB frame is defined by the polarisation vector of the incident photolysis beam $\varepsilon_{p} \| Z$. The CM collision frame is defined by the collision velocity vector $\mathbf{k}\|z\| \mathbf{v}$, the photofragment recoil velocity; for a stationary target, $\mathbf{v}\|\mathbf{k}\| \mathbf{v}_{\mathrm{CM}}$, the centre of mass velocity.

The $\mathrm{LAB}$ angular distribution of the bimolecular reaction products, $\mathrm{AB}, \mathrm{C}$, separating with a relative velocity $\mathbf{k}^{\prime}$ is

$$
\mathrm{I}\left(\hat{\varepsilon}_{p} \cdot \hat{\mathbf{k}}^{\prime}\right) \sim 1+\mathrm{BP}_{2}\left(\hat{\varepsilon}_{p} \cdot \hat{\mathbf{k}}^{\prime}\right)
$$

The coefficient $\mathrm{B}$, reflecting their $\mathrm{LAB}$ translational anisotropy is related to $\beta$, the reagent translational anisotropy, via the equation

$$
\mathrm{B}=\beta\left\langle P_{2}\left(\hat{\mathbf{k}} \cdot \hat{\mathbf{k}}^{\prime}\right)\right\rangle \equiv 5\left\langle P_{2}\left(\hat{\varepsilon}_{p} \cdot \hat{\mathbf{k}}^{\prime}\right)\right\rangle
$$

Determination of the $\mathrm{CM}$ translational alignment $\left\langle P_{2}\left(\hat{\mathbf{k}} \cdot \hat{\mathbf{k}}^{\prime}\right)\right\rangle$ simply involves laboratory frame measurement of the product and reagent translational anisotropies. This can be done by using Doppler resolved laser induced fluorescence (or ionisation) spectroscopy, to probe the reaction products and the recasting of Eq. (2)

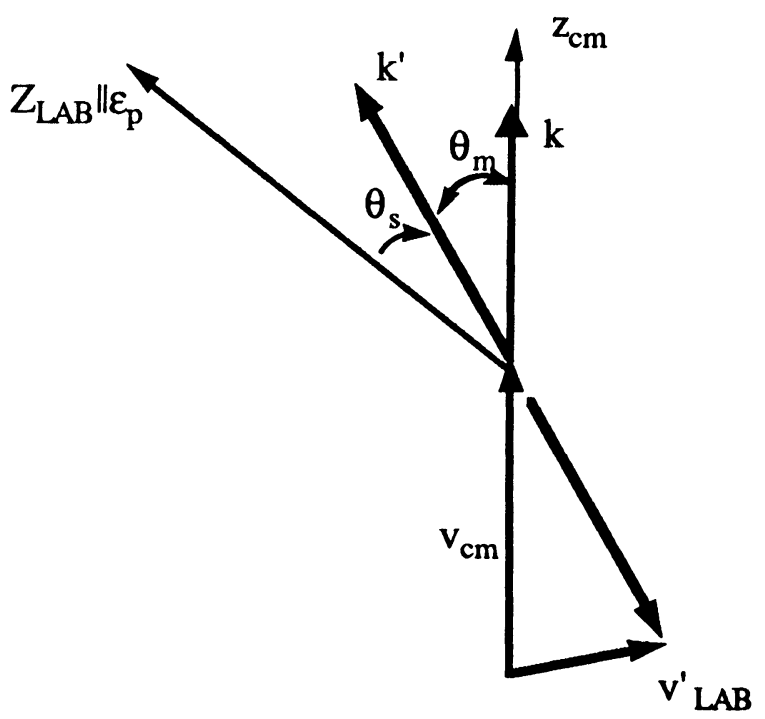

Figure 1 Schematic Newton diagram for the collision of superthermal velocity aligned atoms, with a stationary target molecule. 
in terms of the spectral lineshape function ${ }^{2}$

$$
g(\bar{v}) \sim 1+\beta P_{2}\left(\hat{\varepsilon}_{p} \cdot \hat{\mathbf{k}}_{a}\right) P_{2}\left(\chi_{\mathrm{D}}\right)
$$

$\mathbf{k}_{a}$ represents the probe laser wave vector and $\chi_{\mathrm{D}}$, the relative displacement from the line centre, is defined as

$$
\chi_{\mathrm{D}}=\left(\frac{\bar{v}-\bar{v}}{\bar{v}_{\mathrm{O}}}\right) \frac{c}{v} \equiv \frac{\bar{v}-\bar{v}_{\mathrm{O}}}{\Delta \bar{v}_{\mathrm{D}}}
$$

Rotational alignment. The classical angular distribution of reaction product rotational vectors, $\mathbf{j}$ ' is $\dagger$

$$
\mathrm{I}\left(\hat{\varepsilon}_{p} \cdot \hat{\mathbf{j}}^{\prime}\right) \sim 1+\mathrm{A} P_{2}\left(\hat{\varepsilon}_{p} \cdot \hat{\mathbf{j}}^{\prime}\right)
$$

where

$$
\mathrm{A}=\beta\left\langle P_{2}\left(\hat{\mathbf{k}} \cdot \hat{\mathbf{j}}^{\prime}\right)\right\rangle=5\left\langle P_{2}\left(\hat{\varepsilon}_{p} \cdot \hat{\mathbf{k}}^{\prime}\right)\right\rangle
$$

In terms of the rotational alignment parameter $\mathrm{A}_{O}^{(2)}$, conventionally defined as ${ }^{2,8}$

$$
\mathrm{A}_{O}^{(2)}(\mathrm{LAB}) \equiv 2\left\langle P_{2}\left(\hat{\varepsilon}_{p} \cdot \hat{\mathbf{j}}^{\prime}\right)\right\rangle \quad \text { or } \quad \mathrm{A}_{O}^{(2)}(\mathrm{CM}) \equiv 2\left\langle P_{2}\left(\hat{\mathbf{k}} \cdot \hat{\mathbf{j}}^{\prime}\right)\right\rangle
$$

the alignment coefficient

$$
\mathrm{A} \equiv \frac{5}{2} \mathrm{~A}_{O}^{(2)}(\mathrm{LAB}) \equiv \frac{1}{2} \beta \mathrm{A}_{O}^{(2)}(\mathrm{CM})
$$

in the high $j$ limit.

In practice, estimation of the translational anisotropy and rotational alignment parameters B and A via simulation of the experimental Doppler profiles, requires several levels of convolution, to accommodate the probe laser line profile, the thermal motion of the precursor and target molecules and any spread in the superthermal reagent speeds introduced by the dynamics of the primary photodissociation process. ${ }^{6}$

Other vector correlations. Doppler resolved probing of photoninitiated bi-molecular collision/reaction products can reveal several additional correlations. As with direct photodissociation a total of nine bipolar moments are required to characterise fully the correlated angular distributions of $\mathbf{j}^{\prime}$ and $\mathbf{k}^{\prime}$ about $\varepsilon_{p}$, in a linearly polarised pump-probe experiment. ${ }^{2}$ Provided the reagent translational anisotropy is known, the distributions of $\mathbf{j}^{\prime}$ and $\mathbf{k}^{\prime}$ can be referenced to the $\mathbf{k}$ vector rather than $\varepsilon_{p}$ or the transition dipole $\mu$ and the Doppler profiles can be analysed to obtain the full set of bipolar moments. ${ }^{2}$ Note however, the important correlation $\mathbf{k}^{\prime}, \mathbf{j}^{\prime}$, reflecting angle bending torques at the transition state, is invariant to the LAB $\rightarrow \mathrm{CM}$ transformation.

\section{STEREODYNAMICS OF THE REACTION O $\left({ }^{1} \mathrm{D}\right)+\mathrm{N}_{2} \mathrm{O} \rightarrow \mathrm{NO}+\mathrm{NO}$}

\section{Quantum State Distributions and Scalar Pair Correlations}

Photodissociation of $\mathrm{N}_{2} \mathrm{O}$ at $193 \mathrm{~nm}$ initiates the highly exothermic reaction

$$
\mathrm{O}\left({ }^{1} \mathrm{D}\right)+\mathrm{N}_{2} \mathrm{O} \rightarrow \mathrm{NO}+\mathrm{NO}
$$

$\dagger$ In Eqs. (3) and (7) the factor $\beta / 5$ may be equated with $\left\langle P_{2}\left(\hat{\varepsilon}_{p} \cdot \hat{\mathbf{k}}\right)\right\rangle$, arising from the LAB $\rightarrow \mathrm{CM}$ transformation. 

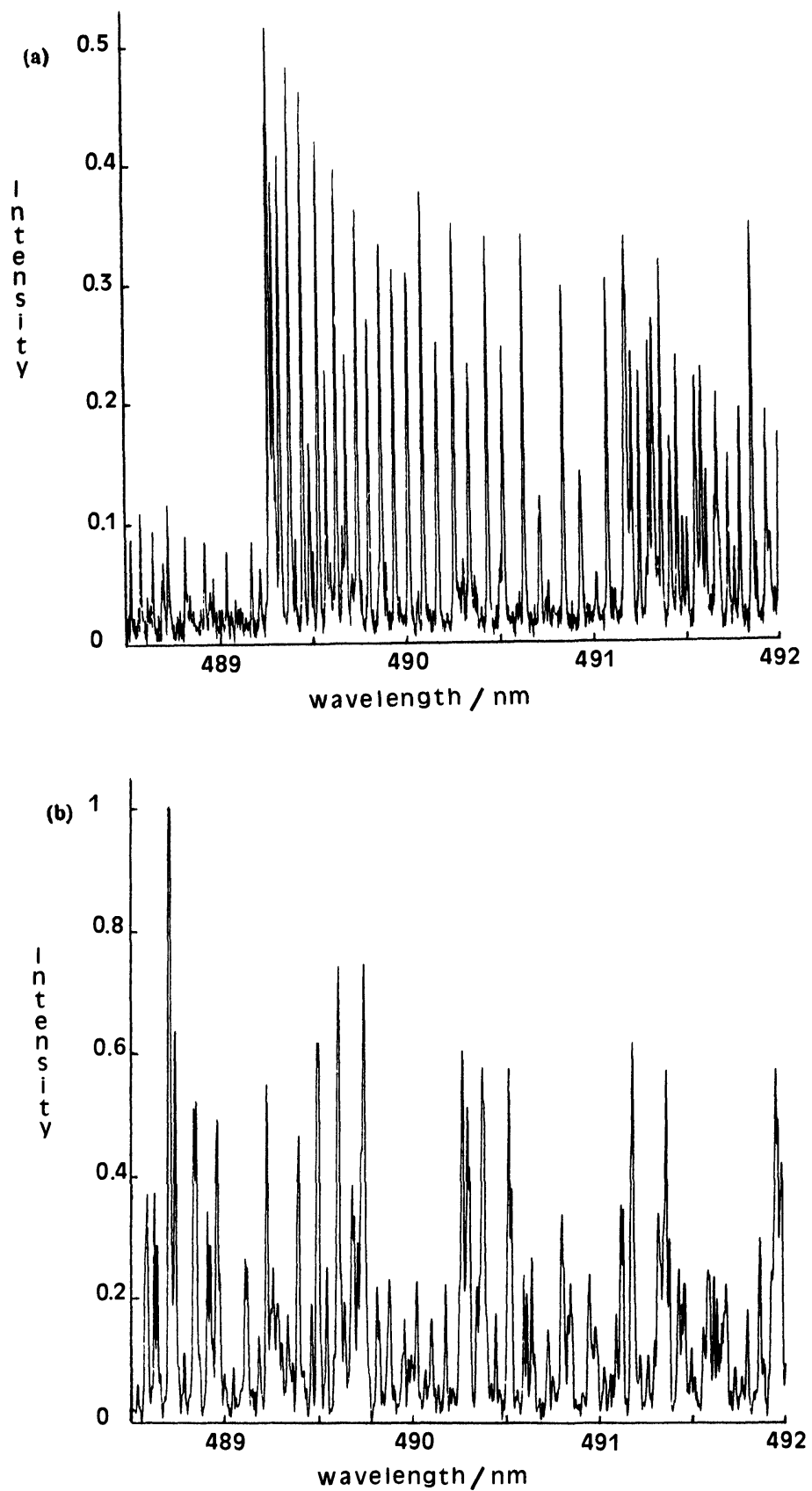

Figure 2 LIF spectra of $\mathrm{NO}(v=17)$ recorded under (a) relaxed, and (b) near single collision conditions. 
which proceeds with near unit collision efficiency to generate NO molecules in a very broad spectrum of rovibrational states up to and including $v_{\mathrm{NO}}=18 .{ }^{7}$ Fully dispersed LIF spectra of NO have been recorded under both single collision and partially relaxed conditions, via the $\gamma(v \leqslant 12)$ and $\beta(10 \leqslant v \leqslant 18)$ band systems. Full assignment of the populated levels is a daunting task, because of the very broad and dense rovibrational product state distributions (see Figure 2) but the task is now largely complete. Apart from those in the level $v=0$ (vide infra) they are all endowed with high levels of rotational excitation (see Figure 2). Population of the most highly excited molecules, i.e. those generated in $v=18$ two quanta above the thermochemical limit, require a collision energy $\left\langle E_{\mathrm{CM}}\right\rangle \leqslant 70 \mathrm{~kJ} \mathrm{~mol}^{-1}$. This can only be introduced via the translational excitation of the reagent $\mathrm{O}\left({ }^{1} \mathrm{D}\right)$ atoms generated in the primary photo-dissociation step.

$$
\mathrm{N}_{2} \mathrm{O}+h v(193 \mathrm{~nm}) \rightarrow \mathrm{N}_{2}(v, \mathrm{~J})+\mathrm{O}\left({ }^{1} \mathrm{D}\right)
$$

If the $\mathrm{N}_{2}$ were formed predominantly in $v_{\mathrm{N}_{2}}=0$, for $\left\langle E_{\mathrm{CM}}\right\rangle \leqslant 70 \mathrm{~kJ} \mathrm{~mol}^{-1}$ energy conservation would require the primary excitation of the $\mathrm{N}_{2}$ into rotational levels $\mathrm{J} \leqslant$ 90 , accounting for $\leqslant 55 \%$ of the available energy. $\dagger$ The analogous photodissociation of the isoelectronic molecule $\mathrm{HN}_{3}$ generates $\mathrm{N}_{2}$ in $v_{\mathrm{N}_{2}}=0$ with $\sim 50 \%$ of the available energy in rotation. ${ }^{9,10}$ Molecules generated in $v_{\mathrm{NO}}=0$ are rotationally cold (mean rotational temperatures $T_{R} \simeq 320 \mathrm{~K}$-see Figure 3) and translationally unexcited (Doppler with $\Delta \bar{v}_{\mathrm{D}}=0.044 \mathrm{~cm}^{-1}$-equivalent to a LAB kinetic energy $\sim 110$ $\mathrm{cm}^{-1}$-see Figure 4). NO molecules in levels $v \geqslant 1$ are rotationally highly excited; e.g. those in $v=1$ have a rotational temperature, $\mathrm{T}_{\mathrm{R}} \sim 5500 \mathrm{~K}$, those in higher vibrational levels appear to be even hotter. They also carry higher translational excitation (see Figure 4). These data allow estimates of the mean energy disposals and by energy conservation, of the scalar pair correlations summarised in Table 1.

The exceptionally cold distribution in $\mathrm{NO}(v=0)$ requires that it be partnered by NO molecules carrying very high levels of internal excitation, predominantly in the "super-excited" levels $16 \leqslant v_{\mathrm{NO}} \leqslant 18$. The narrow spread in the Doppler profiles of $\mathrm{NO}(v=0)$, reflects a narrow spread in the internal energy of the excited partner; the broad distribution over internal rovibrational levels in $\mathrm{NO}(16 \leqslant v \leqslant 18)$ must reflect the spread of collision energies in the hot atom reaction. A "near-stripping" mechanism for the channel leading to $\mathrm{NO}(v=0)$ would accord with the behaviour observed.

The NO molecules produced in levels $v=1-3$ must also be accompanied by highly excited partners but the dramatic jump in their rotational and translational energies suggests the operation of an alternative dynamical pathway. High internal excitation in both NO partners suggests the operation of a short-lived complex mechanism: this view is reinforced by the absence of any measureable translational vector correlation, $\left\langle P_{2}\left(\hat{\mathbf{k}} \cdot \hat{\mathbf{k}}^{\prime}\right)\right\rangle \simeq 0$ (see below).

$\dagger$ A direct measurement of the discussion dynamics by Huber's group gives $\left\langle\left(E_{\text {int }}\left(\mathrm{N}_{2}\right)\right\rangle=58 \%\right.$, and $\beta\left[\mathrm{O}\left({ }^{1} \mathrm{D}\right)\right]=+0.48$ (P. Felder, B.-M. Haas and R. Huber, private communication). 

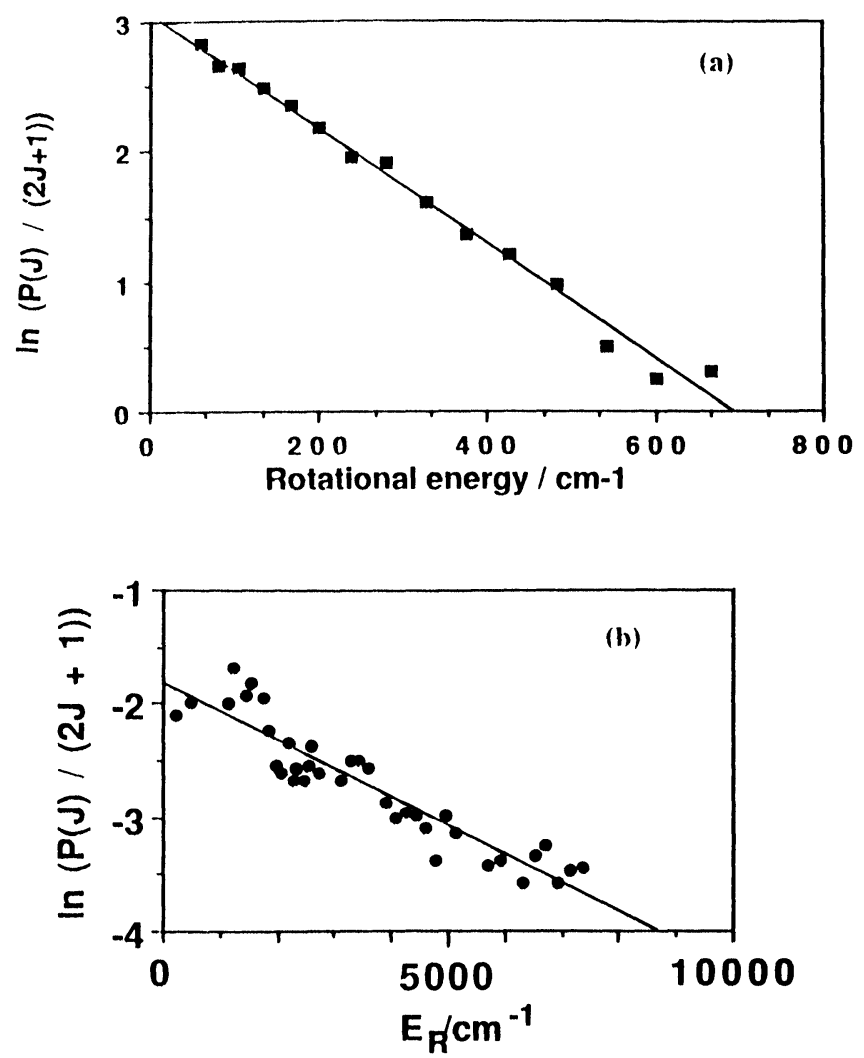

Figure 3 Rotational population distributions in NO; (a) $v=0$; (b) $v=1$.

Vector correlations. The $\mathrm{LAB}$ rotational alignments, $\mathrm{AO}_{O}^{(2)}(\mathrm{LAB})$ for $\mathrm{NO}(v=0)$ are shown in Figure 5; despite the (inevitable) scatter, they are all negative, with $\mathrm{A}_{O}^{(2)}(\mathrm{LAB}) \sim-0.1$. Assuming the product alignment $\left\langle P_{2}\left(\hat{\mathbf{k}} \cdot \hat{\mathbf{j}}^{\prime}\right)\right\rangle>0$, the photofragment alignment must be positive, with $2 \geqslant\langle\beta\rangle \geqslant 1 / 2 . \dagger$ If the cold ("old") $\mathrm{NO}(v=0)$ were a spectator in a pure stripping reaction, no alignment would be expected; there must be some residual interaction between the two NO species at the transition state. The negative alignment could be introduced by reagent orbital angular momentum transfer.

A near stripping mechanism requires $\hat{\mathbf{k}} \cdot \hat{\mathbf{k}}^{\prime} \sim 1$, and a $\mathrm{LAB}$ translational alignment $\mathrm{B}[\mathrm{NO}] \simeq\left\langle\beta\left[\mathrm{O}\left({ }^{1} \mathrm{D}\right)\right]\right\rangle$ (cf. Eq. (3)). The $\hat{\mathbf{k}}, \hat{\mathbf{k}}^{\prime}$ correlation can be estimated from the Doppler profiles; the rotational lines in the slow moving $\mathrm{NO}(v=0)$ are too narrow for the experimental resolution to reveal any changes in line shape when the excitation-detection geometry is changed from coaxial to perpendicular but their

\footnotetext{
$\dagger$ A direct measurement of the discussion dynamics by Huber's group gives $\left\langle\left(E_{\mathrm{int}}\left(\mathrm{N}_{2}\right)\right\rangle=58 \%\right.$, and $\beta\left[\mathrm{O}\left({ }^{1} \mathrm{D}\right)\right]=+0.48$ (P. Felder, B.-M. Haas and R. Huber, private communication).
} 

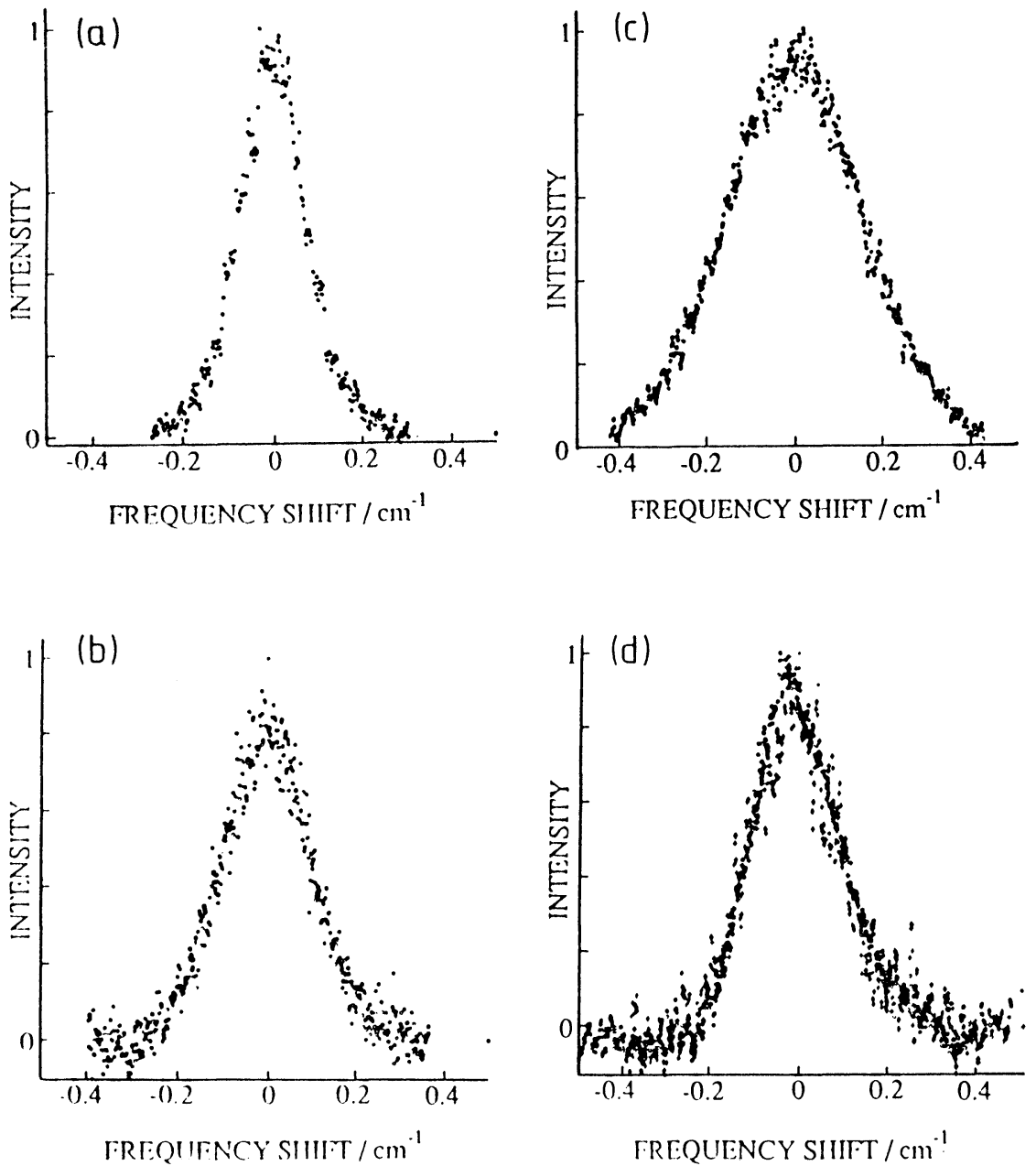

Figure 4 Doppler profiles for $\mathrm{NO}$ (a) $v=0, \mathrm{~N}=20$; (b) $v=1, \mathrm{~N}=35 ;$ (c) $v=3, \mathrm{~N}=31$; all Case A; (d) $v=10, \mathrm{~N}=26$; Case $\mathrm{A}$-circles, Case $\mathrm{D}$-crosses.

Table 1 Energy disposal and pair correlations

\begin{tabular}{|c|c|c|c|c|c|}
\hline & $N O\left(v_{l}\right)$ & $E_{\mathrm{v}} \mathrm{cm}^{-1}$ & $\left\langle E_{R}\right\rangle / \mathrm{cm}^{-1}$ & $\left\langle E_{T}\right\rangle^{L A B} / \mathrm{cm}^{-1}$ & $N O\left(\left\langle v_{2}\right\rangle\right)$ \\
\hline 0 & & 0 & 200 & 110 & $16-18$ \\
\hline 1 & & 1,876 & 4,000 & 690 & 15 \\
\hline 3 & & 5,540 & $>4,000$ & 1,350 & 11 \\
\hline 10 & & 17.600 & not determined & 1,280 & $\leqslant 4$ \\
\hline 15 & & 26,145 & 4.000 & $700^{\mathrm{a}}$ & 1 \\
\hline 16 & & 27,600 & $3-4,000$ & $\geqslant 1,600$ & 0 \\
\hline
\end{tabular}




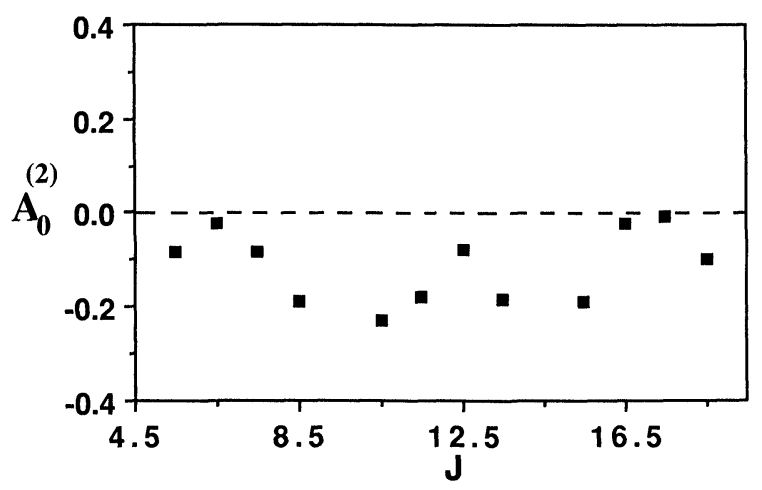

Figure 5 Rotational alignments for $\mathrm{NO}(v=0)$ generated in the reaction of velocity aligned $\mathrm{O}\left({ }^{1} \mathrm{D}\right)$ with $\mathrm{N}_{2} \mathrm{O}$.

forward scattered partners, $\mathrm{NO}(v \sim 16-18)$ are endowed with much higher LAB velocities, $\mathbf{V}_{\mathrm{NO}} \geqslant 1.52 \mathrm{~km} \mathrm{~s}^{-1}$ (for stripping dynamics $\mathbf{V}_{\mathrm{NO}} \geqslant 2 \mathbf{V}_{\mathrm{CM}} \simeq 1.8 \mathrm{~km} \mathrm{~s}^{-1}$ ). The NO molecules excited into intermediate vibrational levels also have larger Doppler widths but their resolved parallel and perpendicular detection profiles are identical within experimental precision (see Figure 4(d)) implying a near isotropic angular distribution, with $\left\langle P_{2}\left(\hat{\mathbf{k}} \cdot \hat{\mathbf{k}}^{\prime}\right)\right\rangle \simeq 0$, and the intermediacy of a short-lived collision complex in the channels leading to vibrationally excited NO pairs.

\section{Acknowledgements}

R. Sayos thanks the British Council and the Spanish Ministry of Education and Science for the award of a Fleming Fellowship, P. A. Enriquez also thanks the Spanish Ministry of Education and Science for postgraduate research support.

\section{References}

1. "Orientation and Polarisation Effects in Reactive Collisions," Faraday Symposium, J. Chem. Soc. Faraday Trans. 2, 1989, 85, 925-1376.

2. R. N. Dixon, J. Chem. Phys. 95, 1866 (1986).

3. J. P. Simons, J. Phys. Chem. 91, 5378 (1987).

4. G. E. Hall and P. L. Houston, Ann. Revs. Phys. Chem. 40, 375 (1989).

5. G. W. Johnston, S. Satyapal, R. Bersohn and B. Katz, J. Chem. Phys. 92, 206 (1990); B. Katz et al. Faraday Discussion Chem. Soc. 91 (1991) in press.

6. F. Green, G. Hancock and A. J. Orr-Ewing, Faraday Discussion Chem. Soc. 91 (1991) in press; F. Green et al. Chem. Phys. Lett. (1991) in press.

7. M. Brouard, S. P. Duxon, P. A. Enriquez, R. Sayos and J. P. Simons, J. Phys. Chem. (1991) in press.

8. R. N. Zare, "Angular Momentum: Understanding Spatial Aspects in Chemistry and Physics," Wiley-Interscience: New York 1988.

9. K.-H. Gericke, R. Theinl and F. J. Comes, J. Chem. Phys. 92, 6548 (1990).

10. J. J. Chu, P. Marcus and P. Dagdigian, J. Chem. Phys. 93, 257 (1990). 